\title{
Study of high-energy neutrino neutral-current interactions
}

\author{
M. Aderholz, ${ }^{\lambda}$ M. M. Aggarwal,,$^{\epsilon}$ H. Akbari, ${ }^{\rho,(a)}$ P. P. Allport, ${ }^{\mu,(b)}$ \\ S. K. Badyal, ${ }^{\kappa}$ H. C. Ballagh, ${ }^{\alpha}$ M. Barth ${ }^{\gamma}$ J. P. Baton,$\xi$ H. H. Bingham, ${ }^{\alpha}$ \\ E. B. Brucker ${ }^{\pi}$ R. A. Burnstein, ${ }^{\imath}$ J. R. Campbell, ${ }^{\theta}$ R. J. Cence, ${ }^{\eta}$ \\ T. K. Chatterjee ${ }^{\epsilon}$ E. F. Clayton ${ }^{\theta}$ G. Corrigan ${ }^{\mu}$ C. Coutures,${ }^{\xi}$ D. DeProspo, ${ }^{\nu}$ \\ Devanand ${ }^{\kappa}$ E. A. De Wolf, ${ }^{\gamma,(\mathrm{c})}$ P. J. W. Faulkner ${ }^{\beta}$ H. Foeth, ${ }^{\delta}$ \\ W. B. Fretter, ${ }^{\alpha,(d)}$ V. K. Gupta, ${ }^{\kappa}$ J. Hanlon,${ }^{\zeta}$ G. Harigel, ${ }^{\delta}$ F. A. Harris, ${ }^{\eta}$ \\ M. A. Jabiol, ${ }^{\xi}$ P. Jacques, ${ }^{\nu}$ V. Jain, ${ }^{\eta,(e)}$ G. T. Jones ${ }^{\beta}$ M. D. Jones,${ }^{\eta}$ T. Kafka, ${ }^{\rho}$ \\ M. Kalelkar, ${ }^{\nu}$ P. Kasper, ${ }^{\xi},(\mathrm{f})$ J. M. Kohli, ${ }^{\epsilon}$ E. L. Koller,${ }^{\pi}$ R. J. Krawiec ${ }^{\beta}$ \\ M. Lauko, ${ }^{\nu}$ J. E. Lys, ${ }^{\alpha}$ P. Marage, ${ }^{\gamma}$ R. H. Milburn, ${ }^{\rho}$ D. B. Miller, ${ }^{\theta}$ I. S. Mittra, ${ }^{\epsilon}$ \\ M. M. Mobayyen, ${ }^{\theta}$ J. Moreels, ${ }^{\gamma}$ D. R. O. Morrison, ${ }^{\delta}$ G. Myatt ${ }^{\mu}$ P. Nailor $,{ }^{\theta},(\mathrm{b})$ \\ R. Naon,$^{\iota}$ A. Napier ${ }^{\rho}$ M. Neveu,${ }^{\xi}$ D. Passmore,${ }^{\rho}$ M. W. Peters,${ }^{\eta}$ \\ V. Z. Peterson, ${ }^{\eta}$ R. Plano, ${ }^{\nu}$ N. K. Rao, ${ }^{\kappa}$ H. A. Rubin, ${ }^{\iota}$ J. Sacton, ${ }^{\gamma}$ \\ S. S. Sambyal,${ }^{\kappa}$ N. Schmitz,${ }^{\lambda}$ J. Schneps,${ }^{\rho}$ J. B. Singh,${ }^{\epsilon}$ W. Smart,${ }^{\zeta}$ \\ P. Stamer, ${ }^{\nu,(\mathrm{g})}$ K. E. Varvell, ${ }^{\beta,(\mathrm{h})}$ L. Verluyten, ${ }^{\gamma,(\mathrm{c})}$ H. Wachsmuth, ${ }^{\delta}$ \\ S. Wainstein, ${ }^{\theta}$ S. Willocq, ${ }^{\gamma,(i)}$ and G. P. Yost ${ }^{\alpha,(j)}$ \\ (E632 Collaboration) \\ ${ }^{\alpha}$ University of California, Berkeley, California 94720 \\ ${ }^{\beta}$ University of Birmingham, Birmingham B15 2TT, United Kingdom \\ ${ }^{\gamma}$ Inter-University Institute for High Energies, B-1050 Brussels, Belgium \\ ${ }^{\delta}$ CERN, CH-1211, Geneva 23, Switzerland \\ ${ }^{\epsilon}$ Panjab University, Chandigarh-160014, India \\ ${ }^{\zeta}$ Fermilab, Batavia, Illinois 60510 \\ ${ }^{\eta}$ University of Hawaii, Honolulu, Hawaii 96822 \\ ${ }^{\theta}$ Imperial College of Science and Technology, London SW7 2AZ, United Kingdom \\ 'Mlinois Institute of Technology, Chicago, Ilinois 60616 \\ ${ }^{\kappa}$ University of Jammu, Jammu-180001, India \\ ${ }^{\lambda}$ Max-Planck-Institut fur Physik und Astrophysik, D-8000 Munchen 40, Germany \\ " Department of Nuclear Physics, University of Oxford, Oxford OX1 3RH, United Kingdom \\ ${ }^{\nu}$ Rutgers University, New Brunswick, New Jersey 08903 \\ ${ }^{\xi}$ Departement de Physique des Particules Elementaires, Centre d'Etudes Nucleaires, Saclay, \\ F-91191 Gif-sur-Yvette, France \\ ${ }^{\pi}$ Stevens Institute of Technology, Hoboken, New Jersey 07030 \\ ${ }^{\circ}$ Tufts University, Medford, Massachusetts 02155
}

(Received 28 June 1991)

From an exposure of the Fermilab 15-foot bubble chamber to the Tevatron quadrupole triplet neutrino beam, we have determined the ratio of neutral-current (NC) to charged-current (CC) interactions to be $0.288 \pm 0.032$ for events with visible hadron momentum above $10 \mathrm{GeV} / c$. The mean $\nu(\bar{\nu})$ event energy is $150(110) \mathrm{GeV}$, which is higher than that for any previous beam. This result agrees with those from previous experiments at lower energies. The NC/CC ratio is derived for a combined sample of $\nu$ and $\bar{\nu}$ events. A value of $0.274 \pm 0.038$ is obtained for the dominant $\nu$ component assuming $\bar{\nu} \mathrm{NC} / \mathrm{CC}=0.39 \pm 0.08$. For events with visible hadron momentum above $25 \mathrm{GeV} / c$, where the neutral hadron contamination remaining in the $\mathrm{NC}$ sample is assumed to be negligible, the combined $\mathrm{NC} / \mathrm{CC}$ is $0.323 \pm 0.025$ and the $K^{0}$ production rates are $0.375 \pm 0.064$ per $\mathrm{CC}$ and $0.322 \pm 0.073$ per $\mathrm{NC}$ event. The corresponding $\Lambda$ rates are $0.161 \pm 0.030$ per $\mathrm{CC}$ and $0.113 \pm 0.030$ per NC event. The $K^{0}$ and $\Lambda$ distributions of the fractional hadron energy variable $z$ in $\mathrm{NC}$ events are consistent with those in $\mathrm{CC}$ events.

PACS number(s): 13.15.Jr

(a) Now at Johns Hopkins University, Baltimore, MD 21218.

(b) Now at Cavendish Laboratory, Cambridge, U.K.

(c) Also at Universitaire Instelling Antwerpen, B-2610 Wilrijk, Belgium.

(d) Deceased.

(e) Now at Syracuse University, Syracuse, NY 13210. (f) Now at Fermilab, Batavia, IL 60510

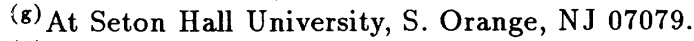

${ }^{(h)}$ Current address: 20 Prince St., Queens Park, W. Australia 6107.

(i) Now at Tufts University, Medford, MA 02155.

(j) Now at SSC Laboratory, Dallas, Texas 75237 . 


\section{INTRODUCTION}

Studies of neutral-current (NC) neutrino interactions allow tests of the standard model of electroweak interactions and determination of the parameter $\sin ^{2} \theta_{W}$. Some recent reviews are given in Refs. [1-5]. The main motivation for studying neutrino interactions using the Tevatron at Fermilab is to test the standard model and to look for new phenomena at energies above those accessible to previous neutrino experiments. The higher neutrino energies also produce a smaller fraction of charged-current (CC) events with low-energy muons; thus, the muon detection efficiency improves and the contamination of unidentified $\mathrm{CC}$ events in the NC sample is reduced. An additional advantage is that new Internal Picket Fence (IPF) counters were installed around the Fermilab 15 -foot bubble chamber for better determination of event times and a new External Muon Identifier (EMI) was built for improved muon identification. This experiment (E632) is the first to use this improved apparatus.

This paper reports results from a study of $\mathrm{NC}$ events from about $10 \%$ of the film taken for E632 in 1985. Section II contains a description of the beam, running conditions, and the detectors used. Section III discusses muon identification necessary to identify $\mathrm{CC}$ events induced by muon neutrinos and estimates hadron misidentification. Section IV discusses the criteria used to extract a sample of $\mathrm{NC}$ events and to remove electron-neutrino $\mathrm{CC}$ events and neutral hadron-induced events. The remaining backgrounds and losses in this sample are calculated to determine the ratio NC/CC. Section V compares strange-particle production in $\mathrm{NC}$ and $\mathrm{CC}$ events. Finally, Sec. VI summarizes the results.

\section{EXPERIMENTAL CONDITIONS}

Experiment E632 took data both in the 1985 and in the 1987 running periods for fixed target experiments at Fermilab. This paper reports only on data from the 1985 run. (Measurements and analysis are in progress on data from the 1987 run which, because of the lighter mixture in the bubble chamber, is better suited to studies of minimum-bias event samples.) The neutrino beam was produced by the quadrupole triplet train (tuned to a momentum of $300 \mathrm{GeV} / c$ ), which focused secondaries produced by interactions of $800-\mathrm{GeV}$ protons from the Tevatron. In the fiducial volume $\left(15 \mathrm{~m}^{3}\right)$ of the 15 -foot bubble chamber, $\nu$ and $\bar{\nu}$ CC events are produced in a ratio of about 6 to 1 and with mean energies of 150 and $110 \mathrm{GeV}$, respectively. About $30 \%$ of $\nu$ and $14 \%$ of $\bar{\nu}$ events have energies above $200 \mathrm{GeV} ; 9 \%$ of $\nu$ and $2 \%$ of $\bar{\nu}$ events have energies above $300 \mathrm{GeV}$, a region inaccessible to pre-Tevatron experiments. Less than $1 \%$ of the events have energies above $500 \mathrm{GeV}$.

In the 1985 run, the 15 -foot bubble chamber was filled with a $75 \%-25 \%$ molar neon-hydrogen mixture giving a density of $0.70 \mathrm{~g} / \mathrm{cm}^{3}$. The chamber was photographed by three conventional cameras, one high resolution camera, and a camera used to record holograms. The Internal Picket Fence (divided into an upstream half IPF $\mathrm{A}$ and a downstream half IPF B) and three planes of the External Muon Identifier (EMI A, B, and C) were positioned around the chamber as shown in Figs. 1 and 2. More details about the IPF and EMI are given in our paper on dimuon events Ref. [6].

On about $10 \%$ of the film from the 1985 run, all events which were not clearly associated with interactions in material upstream of the chamber and which had visi-

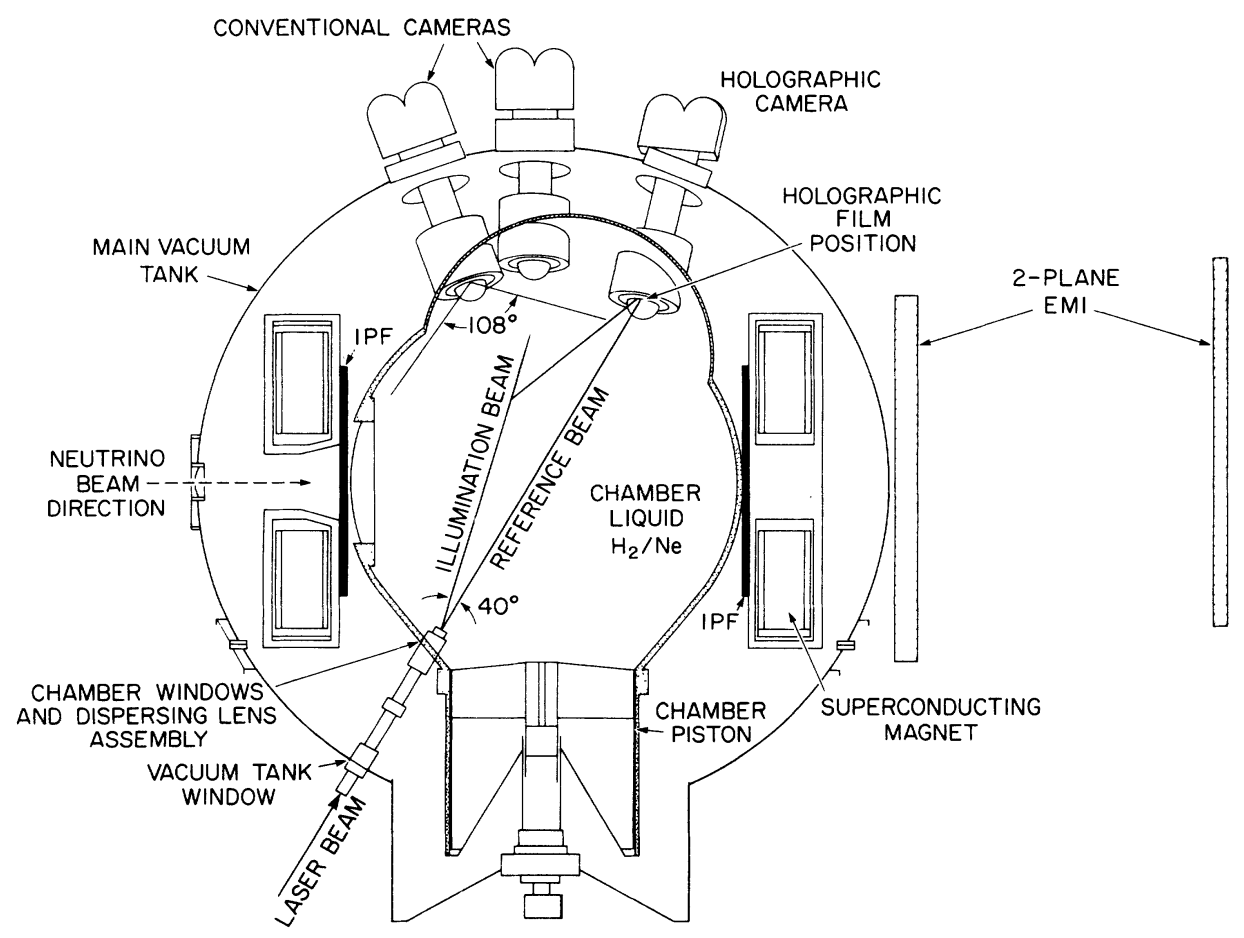

FIG. 1. Side view of the 15-foot bubble chamber. The positions of the IPF and the EMI B and C planes are shown. 


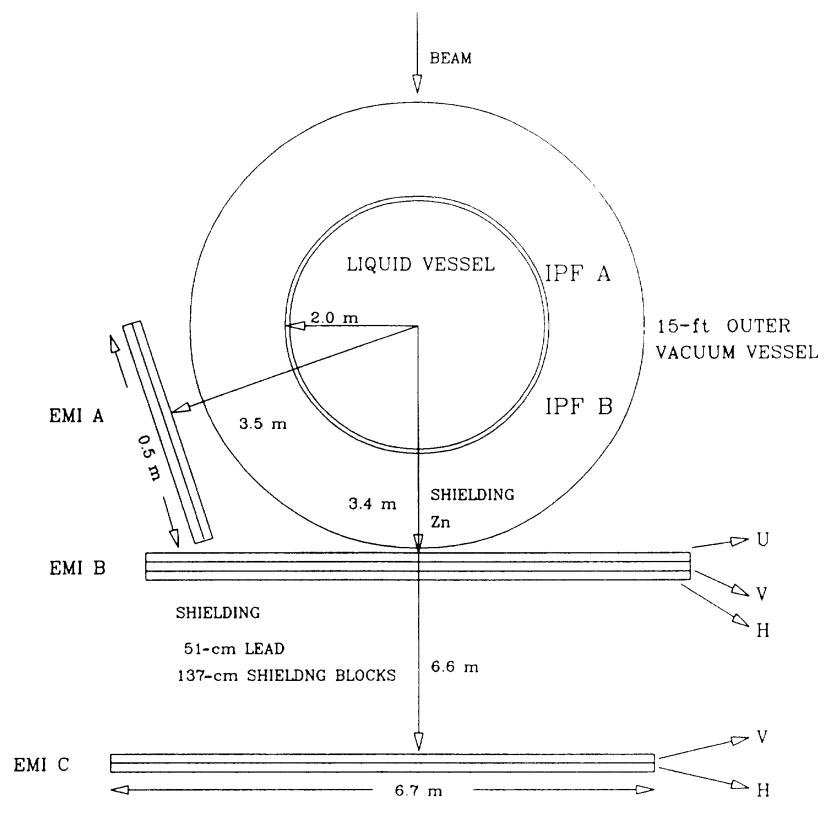

FIG. 2. Top view of the IPF and EMI. Not to scale.

ble longitudinal momentum (i.e., momentum projected onto the neutrino beam direction) above $5 \mathrm{GeV} / c$ were measured. Frames for which there was no EMI/IPF data were excluded from this study. The scanning efficiency for CC events with at least two prongs was determined from double scanning to be 0.99 after the two scans. The efficiency for events with visible hadronic momentum above $10 \mathrm{GeV} / c$ is slightly larger; we have checked that the scanning efficiencies are above 0.99 for both $\mathrm{NC}$ and $\mathrm{CC}$ events with at least two prongs and visible hadronic momentum above $10 \mathrm{GeV} / c$. (Note that these efficiencies cancel in taking the ratio NC/CC.) About $2 \%$ of the events were so complex that they were measured only partially; these partial measurements included tracks of any possible muon candidates. The fraction of events that were so complex that tracks of possible muon candidates could not be measured reliably was less than $0.5 \%$. Partially measured events are used for this analysis; the unmeasurable events are excluded.

The general event measurements included all tracks from the primary neutrino vertex and all $V^{0}$ 's (possible neutral-strange-particle decays) associated with the primary vertex. Outgoing tracks from interactions on primary tracks were not usually measured. Neutral-particle interactions associated with the primary vertex were required to be measured only if their visible momentum exceeded $5 \mathrm{GeV} / c$. Only the five gamma conversions closest to the primary vertex were required to be measured. Applying these criteria to events generated by a Monte Carlo program that used the expected neutrino flux and simulated measured events indicates that, on average, about $70 \%$ of the total hadronic energy will be measured. This bias in the hadronic energy measurement is expected to be the same for $\mathrm{NC}$ and CC events because the same measurement criteria were applied.

\section{MUON IDENTIFICATION}

Muon identification relies upon the EMI so events for which there is no acceptable EMI data are excluded from this analysis. Each measured track which traverses the bubble chamber without interacting is extrapolated to predict which tubes in the EMI planes would be hit if the track was made by a muon. There are 3 to 5 hadronic interaction lengths of absorber between the chamber and the EMI B plane plus another 4 to 6 interaction lengths between the EMI B and C planes. For each track predicted to hit the EMI, a matching algorithm looks for a time slot for which there are horizontal and vertical tubes hit close to those predicted. (The EMI B plane also has a diagonal set of tubes, which are labeled $U$ in Fig. 2.) For a time slot where there are close matches in both the EMI B and $\mathrm{C}$ planes for the $\mathrm{H}$ and $\mathrm{V}$ tubes, a correlated $\chi^{2}$ for the quality of the match is calculated. Because the error in the predicted position (due to measurement errors and multiple Coulomb scattering in the absorber) for high-momentum muons is smaller than the tube width, this calculated $\chi^{2}$ does not have the usual distribution. For an event to be in the CC sample, we require at least one track to have a match in the EMI $\mathrm{B}$ and $\mathrm{C}$ planes with a correlated $\chi^{2}$ value less than 13 . No explicit cut is made on the momentum of identified muons but less than $10 \%$ of muons with momentum below $4 \mathrm{GeV} / c$ are predicted to hit EMI C. Only one event in the CC sample has a muon with momentum below 4 $\mathrm{GeV} / c$.

Electronic inefficiencies in the EMI can result in some muons matching with hits in only 3 of the $4(\mathrm{H}$ and $\mathrm{V})$ coordinates in EMI B and C. The efficiency of the EMI to identify muons is determined by applying the muon identification criteria to measured beam tracks with momenta above $20 \mathrm{GeV} / c$. (Beam tracks are tracks entering the upstream half of the chamber in a direction close to the neutrino beam direction and traversing the chamber without interacting. In order to determine the EMI electronic efficiency using beam tracks, they are all assumed to be muons.) The resulting efficiencies are given in Table I.

We define a separate category for events with a threecoordinate muon in order to remove them from the $\mathrm{NC}$ sample but do not include them in the CC sample because these events do include some real $\mathrm{NC}$ events in which a hadron leaving the bubble chamber is misidentified. To keep the contribution from misidentified hadrons small, we require 3 -coordinate muons to have momentum above $4 \mathrm{GeV} / c$. Corrections for the loss from the CC sample and for that from the $\mathrm{NC}$ sample are discussed below. Another type of $\mathrm{CC}$ event that is a potential background in the NC sample is one in which the muon misses the EMI C plane. This is a significant problem only for $\nu$ induced CC events because of the smaller number of $\bar{\nu}$ induced events. Therefore, we also exclude from both the $\mathrm{CC}$ and $\mathrm{NC}$ samples events in which a negatively charged leaving track is predicted to miss EMI C, makes good matches with hits in the $\mathrm{V}$ and $\mathrm{H}$ tubes in EMI B, and has momentum above $4 \mathrm{GeV} / c$. Also, for these events and for those with a 3 -coordinate muon, we require some 
TABLE I. EMI acceptances determined from Monte Carlo events and EMI electronic efficiencies determined using measured beam tracks.

\begin{tabular}{|c|c|c|c|c|}
\hline \multirow{2}{*}{ Muon momentum } & \multicolumn{2}{|c|}{$>4 \mathrm{GeV} / c$} & \multicolumn{2}{|r|}{$<4 \mathrm{GeV} / \mathrm{c}$} \\
\hline & $\mu^{-}$ & $\mu^{+}$ & $\mu^{-}$ & $\mu^{+}$ \\
\hline \multirow[t]{2}{*}{ Fraction of all CC events } & 0.956 & 0.985 & 0.044 & $\overline{0.015}$ \\
\hline & \multicolumn{4}{|c|}{ fraction of muons in each momentum region } \\
\hline Muon hits both EMI planes & 0.965 & 0.976 & 0.066 & 0.025 \\
\hline Muon hits only EMI B (inner plane) & 0.031 & 0.023 & 0.342 & 0.292 \\
\hline Muon misses both EMI planes & 0.004 & 0.001 & 0.592 & 0.683 \\
\hline \multicolumn{5}{|c|}{ EMI efficiencies for muons hitting both EMI planes (from 2520 beam tracks) } \\
\hline Fraction with 4-coordinate matches & & & & $=0.947 \pm 0.004$ \\
\hline Fraction with 3 -coordinate matches & & & & $=0.044 \pm 0.004$ \\
\hline Fraction with matches in both $\mathrm{V}$ and $\mathrm{H}$ coord. in EMI B & & & & $=0.972$ \\
\hline
\end{tabular}

hits in the downstream IPF (IPF B) at the muon time slot determined by the EMI.

To determine the acceptance of the EMI for muons from CC events, we use samples of about $8000 \nu$ and $8000 \bar{\nu}$ CC events generated by a Monte Carlo program that uses the expected flux distributions (calculated using the parameters of the quadrupole triplet beam by the program NUADA as in Ref. [6]) and which simulates measured events in the bubble chamber. These simulated events are then processed with the same programs used for real events. The results for the EMI acceptances are given in Table I. These acceptances are essential to calculate the expected CC contamination in the NC sample and to correct the observed number of CC events for losses due to muons that miss one or both EMI planes.

Using the acceptances and efficiencies given in Table I, one can calculate the fractions of $\nu$ and $\bar{\nu}$ CC events expected to satisfy the muon identification criteria. The results are summarized in Table II. Note that most of the undetected negatively charged muons have momenta below $4 \mathrm{GeV} / c$. Also, although the fractions of undetected muons are about the same for $\nu$ and $\bar{\nu}$ events, the main contribution to the CC contamination in the NC sample comes from $\nu$ events because of the larger $\nu$ flux and cross section.

Some NC events are lost from the NC sample because a hadron is misidentified as a muon. These misidentified hadrons are usually classified as 3-coordinate muons and only rarely as 4-coordinate muons so most of the events lost from the NC sample will not be in the CC sample.
This loss is estimated by looking at events in the CC sample for which more than one track satisfies the muon identification criteria corresponding to distinct hits in EMI C. Real dimuon events (as defined in Ref. [6]) are excluded from this study. The loss from the NC sample due to hadrons misidentified in any of the muon classifications depends upon the hadronic energy. The fractional loss is $0.049 \pm 0.007$ for visible hadronic momentum above 10 $\mathrm{GeV} / c$ and $0.065 \pm 0.009$ for momentum above $25 \mathrm{GeV} / c$. The NC contamination fraction in the CC sample (4coordinate muon) is estimated to be $0.0068 \pm 0.0013$.

\section{SELECTION OF THE NC SAMPLE AND EXTRACTION OF THE NC SIGNAL}

\section{A. Contamination in the NC sample}

The muon identification criteria specified above identify most $\mathrm{CC}$ events induced by muon neutrinos and leave only a small contamination in the NC sample. A second source of contamination in the NC sample is CC events induced by electron neutrinos. This contamination is smaller than that for muon neutrinos because of the much smaller flux of electron neutrinos and because electron neutrino CC events can be identified by the presence of a single, high-energy electron from the primary interaction. Events with identified primary electrons are removed from the NC sample. In the fraction

TABLE II. Muon detection efficiencies based upon the EMI acceptances and efficiencies in Table I and including an IPF efficiency of 0.93 for 3 -coordinate and EMI B only muons.

\begin{tabular}{|c|c|c|c|c|}
\hline \multirow[t]{2}{*}{ Muon momentum } & \multicolumn{2}{|c|}{$>4 \mathrm{GeV} / c$} & \multicolumn{2}{|c|}{$<4 \mathrm{GeV} / c$} \\
\hline & $\mu^{-}$ & $\mu^{+}$ & $\mu^{-}$ & $\mu^{+}$ \\
\hline \multirow[t]{2}{*}{ Fraction of all CC events } & 0.956 & 0.985 & 0.044 & 0.015 \\
\hline & \multicolumn{4}{|c|}{ fraction of muons detected in each momentum region } \\
\hline Muon detected by 4-coord. match & 0.914 & 0.924 & 0.063 & 0.024 \\
\hline Muon detected by 3-coord. match & 0.040 & 0.040 & - & - \\
\hline Muon detected only by EMI B & 0.028 & - & - & - \\
\hline Muon misses both EMI planes & 0.000 & 0.000 & 0.000 & 0.000 \\
\hline Undetected fraction of muons in momentum region & 0.018 & 0.036 & 0.937 & 0.976 \\
\hline \multicolumn{5}{|c|}{ Total undetected $\mu^{-}$fraction $=(0.956)(0.018)+(0.044)(0.937)=0.0585$} \\
\hline \multicolumn{5}{|c|}{ Total undetected $\mu^{+}$fraction $=(0.985)(0.036)+(0.015)(0.976)=0.0489$} \\
\hline
\end{tabular}


(about $\frac{1}{3}$ ) of events examined carefully for primary electrons of energy above $4 \mathrm{GeV}$, for which our estimated detection efficiency is $0.80 \pm 0.10$, events with identified primary electrons correspond to $3.4 \%$ of events with detected muons. Correcting for detection efficiency gives a rate for electron neutrino events which is $0.044 \pm 0.010$ times that for muon neutrino events. Events with undetected primary electrons in the entire NC sample give a contamination which is about $40 \%$ of that from events with undetected muons. Most of this electron-neutrino contamination comes from the $\frac{2}{3}$ of the sample for which the estimated electron detection efficiency is only $28 \%$ because there was no special scan for primary electrons.

A third source of contamination in the NC sample is neutral-hadron-induced events. Neutral hadrons are produced by neutrino interactions upstream of the chamber and generally have momenta of a few $\mathrm{GeV} / c$. Therefore, most of this contamination is removed by the requirement of at least $5 \mathrm{GeV} / c$ visible momentum. The visible momentum spectrum of neutral-hadron-induced events associated with neutrino interactions is shown in Fig. 3. We assume that, for momenta above $5 \mathrm{GeV} / c$, the spectrum of neutral hadrons entering the chamber is the same as that in Fig. 3. The main source of hadrons entering the chamber is neutrino interactions in the magnet coils, which are the only large quantity of dense matter within $30 \mathrm{~m}$ upstream of the chamber. Hadrons produced in these neutrino interactions rarely have momenta above $25 \mathrm{GeV} / c$ so they are unlikely to produce neutral hadrons above $5 \mathrm{GeV} / c$ if they interact before exiting the coils. Therefore, it is plausible that most incoming neutral hadrons above $5 \mathrm{GeV} / c$ are directly produced in neutrino interactions in the coils.

The main quantities used to associate neutral-induced

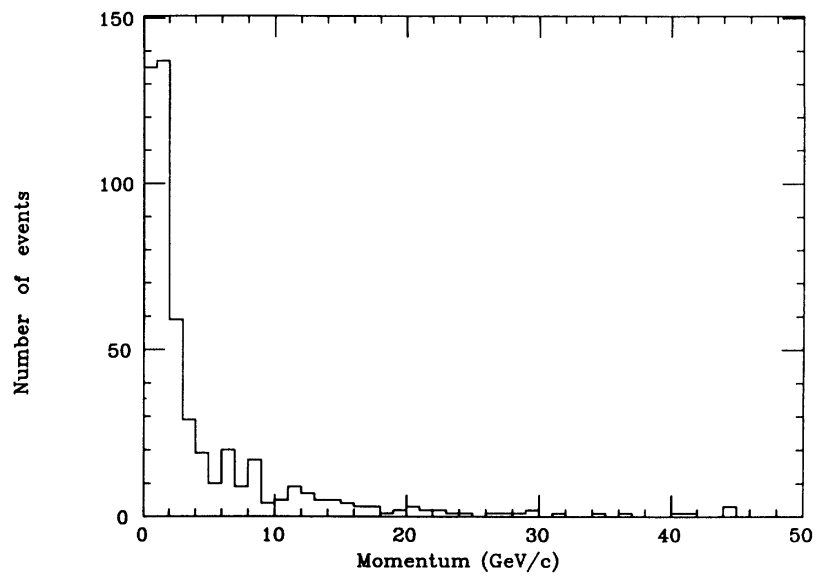

FIG. 3. Momentum spectrum of neutral-hadron-induced events associated with neutrino interactions.

events to upstream events are the longitudinal and transverse projections of the visible momentum of the downstream event relative to the direction defined by the two vertices. If the distance between the vertices is less than $20 \mathrm{~cm}$, the only requirement is that the longitudinal momentum exceed the transverse momentum. For vertices separated by more than $20 \mathrm{~cm}$, the transverse momentum must be less than an upper limit which can vary between 0.6 and $5.0 \mathrm{GeV} / c$. The upper limit is chosen from the larger of the following quantities: (1) estimated error on the transverse momentum; and (2) $10 \%$ of the total momentum of the downstream event, provided that this value is between 0.6 and $2.0 \mathrm{GeV} / c$. Note that, of the events associated by these criteria, the fraction which have visible momenta above $25 \mathrm{GeV} / c$ is $2.6 \%$ of the total and $10 \%$ of those with visible momenta above $5 \mathrm{GeV} / c$.

TABLE III. Numbers of observed events, expected backgrounds, and corrected numbers of NC and $\mathrm{CC}$ events for different regions of visible hadron momentum. The value of NC/CC for momentum $>25 \mathrm{GeV} / c$ is calculated assuming that the neutral hadron background is negligible after removing the 21 events with associated IPF A hits. The resulting value of NC/CC is applied to $\mathrm{CC}$ events with hadron momenta in the $5-10 \mathrm{GeV} / c$ region to predict the number of NC events in this region. Subtracting this predicted number of NC events and the expected CC background from the $220 \mathrm{NC}$ candidates gives the number of neutral-hadron-induced events in this momentum region. The expected neutral hadron background in the higher momentum regions is then derived from that in the $5-10 \mathrm{GeV} / c$ region using the observed momentum distribution (Fig. 3 ) of neutral-hadron-induced events associated with neutrino interactions.

\begin{tabular}{lcr}
\hline \hline & Observed & Expected \\
\hline 4-coord. $\mu^{-}$ & 1492 & \\
4-coord. $\mu^{+}$ & 249 & \\
& & \\
3-coord. $\mu^{-}$ & 62 & $12.7 \pm 0.8 \pm 1.7$ \\
3-coord. $\mu^{+}$ & 15 & $46.0 \pm 1.2$ \\
& & $(5.7 \pm 0.4)$ \\
EMI B only $\mu^{-}$ & 31 & $94.6 \pm 2.5$ \\
EMI B only $\mu^{+}$ & - & $12.7 \pm 0.8$ \\
& & \\
Undetected $\mu^{-}$ & & \\
Undetected $\mu^{+}$ & & \\
Primary $e^{-}(>4 \mathrm{GeV})$ & 29 & \\
Primary $e^{+}(>4 \mathrm{GeV})$ & 8 & \\
\hline
\end{tabular}


TABLE III. (Continued).

\begin{tabular}{|c|c|c|}
\hline & Observed & Expected \\
\hline Undetected $e^{+-}$ & & $39.3 \pm 9.3$ \\
\hline \multicolumn{3}{|c|}{ Events with visible hadron longitudinal momentum from $5-10 \mathrm{GeV} / c$} \\
\hline 4-coord. $\mu^{-}+\mu^{+}$ & $147+37$ & \\
\hline $\mathrm{NC}$ candidates & 220 & \\
\hline IPF A assoc. & 52 & \\
\hline Expected CC background & & $2.5 \pm 0.1$ \\
\hline Expected NC signal & & $59.2 \pm 6.5$ \\
\hline \multicolumn{3}{|c|}{ subtracting $\mathrm{CC}$ bkgd $+\mathrm{NC}$ signal } \\
\hline$\rightarrow$ neutral hadron background & & $158.3 \pm 16.2$ \\
\hline \multicolumn{3}{|c|}{ Events with visible hadron longitudinal momentum $>10 \mathrm{GeV} / c$} \\
\hline 4-coord. $\mu^{-}+\mu^{+}$ & $1187+172$ & \\
\hline $\mathrm{NC}$ candidates & 742 & \\
\hline IPF A assoc. & 72 & \\
\hline Undetected $\mu^{+-}$ & & $102.9 \pm 2.5$ \\
\hline Undetected $e^{+-}$ & & $37.7 \pm 8.9$ \\
\hline Neutral hadron background & & $174.1 \pm 36.3$ \\
\hline$\rightarrow$ NC signal & & $427.3 \pm 46.3$ \\
\hline Corrected $\mathrm{NC}$ & & $461.3 \pm 50.0$ \\
\hline Corrected CC & & $1600.8 \pm 46.1$ \\
\hline $\mathrm{NC} / \mathrm{CC}$ & & $0.288 \pm 0.032$ \\
\hline \multicolumn{3}{|c|}{ Events with visible hadron longitudinal momentum $>25 \mathrm{GeV} / c$} \\
\hline 4-coord. $\mu^{-}+\mu^{+}$ & $812+103$ & \\
\hline NC candidates & 458 & \\
\hline IPF A assoc. & 21 & \\
\hline Undetected $\mu^{+-}$ & & $85.0 \pm 2.1$ \\
\hline Undetected $e^{+-}$ & & $31.1 \pm 7.4$ \\
\hline Neutral hadron background & & $(34.4 \pm 11.0)$ \\
\hline$\rightarrow \mathrm{NC}$ signal & & $320.9 \pm 22.3$ \\
\hline Corrected NC & & $348.1 \pm 24.2$ \\
\hline Corrected CC & & $1078.4 \pm 37.1$ \\
\hline $\mathrm{NC} / \mathrm{CC}$ & & $0.323 \pm 0.025$ \\
\hline
\end{tabular}

The upstream IPF (IPF A) can be used to remove most high-momentum neutral-hadron-induced events from the $\mathrm{NC}$ sample. For a visible longitudinal momentum above $25 \mathrm{GeV} / c$, we reject events for which there are three or more IPF A tubes within a cone of half-angle 120 milliradians upstream of the event vertex (see Appendix) which have hits in the event time slot. Assuming that the remaining neutral hadron contamination above $25 \mathrm{GeV} / c$ is negligible, we subtract the expected CC contamination to get the true number of NC events detected. Table III summarizes the observed numbers of events and expected backgrounds. Figure 4 shows the visible longitudinalmomentum distribution for the $\mathrm{NC}$ candidates that are not rejected by IPF A. For comparison, Fig. 5 shows the visible longitudinal hadron momentum distributions for events in the CC sample.

\section{B. Corrections for losses of NC events}

Because of the low scanning efficiency for events with fewer than two prongs, one needs to correct the NC sample for this loss. Note that all CC events have at least one prong and that the only 1-prong events are $\bar{\nu}+$ proton interactions in which all of the produced hadrons are neutral. These events are very rare, especially for visible hadron momentum above $10 \mathrm{GeV} / c$. (A special scan using the EMI to predict positions of events producing muons was done as an independent check of our efficiency 


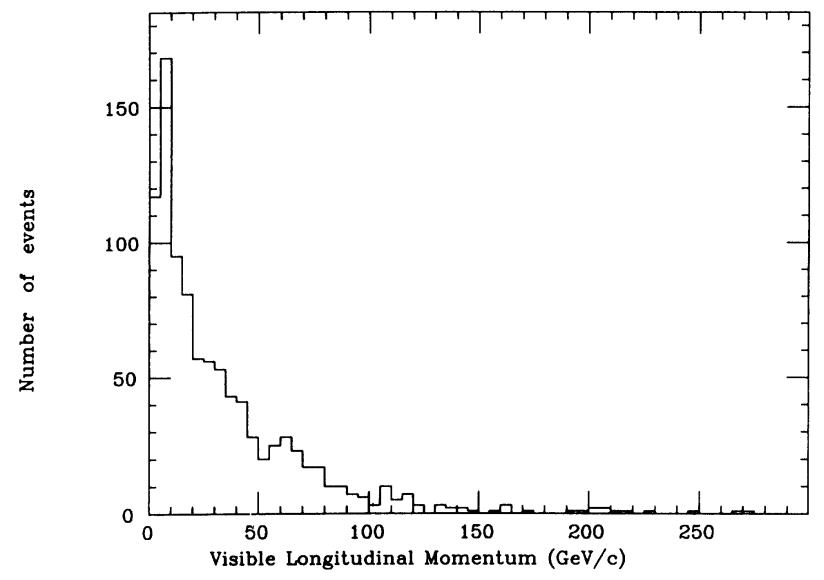

FIG. 4. Visible longitudinal momentum of NC candidates. Events vetoed by IPF A are excluded from this plot.

for finding low-multiplicity $\mathrm{CC}$ events.) To estimate the loss of 0 -prong and 1-prong NC events, we use 1-prong and 2-prong CC events. The total loss is $2.6 \%$ in the region above $10 \mathrm{GeV} / c$ and $1.4 \%$ above $25 \mathrm{GeV} / c$.

The other mechanism for loss of $\mathrm{NC}$ events is misidentification of hadrons as muons. This loss depends upon the momentum of the hadron system and is estimated using hadrons in CC events. (See Sec. III.) The corrected numbers of $\mathrm{NC}$ and $\mathrm{CC}$ events (for the same cut on visible hadron momentum) are given in Table III. Because the corrected number of $\mathrm{NC}$ events includes those induced by electron neutrinos, the corrected number of muon neutrino CC events is multiplied by $1.044 \pm 0.010$ to include the contribution from electron neutrinos.

\section{Calculation of $\mathrm{NC} / \mathrm{CC}$}

Assuming that the only neutral hadron contamination in the $458 \mathrm{NC}$ candidates are the 21 events with

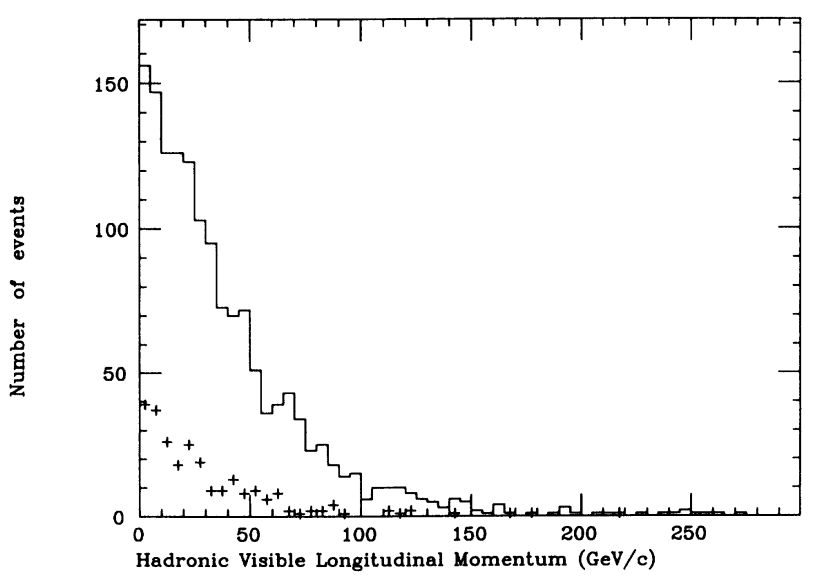

FIG. 5. Hadronic visible longitudinal momentum of $\mathrm{CC}$ events. The histogram is the data for events with an identified $\mu^{-} ;$the distribution indicated with plusses is for events with an identified $\mu^{+}$. associated IPF A hits and dividing the corrected numbers of $\mathrm{NC}$ and $\mathrm{CC}$ events gives a value of $\mathrm{NC} / \mathrm{CC}$ of $0.323 \pm 0.025$ for visible longitudinal hadron momentum above $25 \mathrm{GeV} / c$. Using this value of $\mathrm{NC} / \mathrm{CC}$ one can calculate the number of true $\mathrm{NC}$ events in the $5-10 \mathrm{GeV} / c$ region. Subtracting from the number of observed events in this momentum region shows that most of the observed events are due to neutral hadron interactions. Changing the assumed $\mathrm{NC} / \mathrm{CC}$ value by 0.1 would change the calculated neutral hadron background in the $5-10 \mathrm{GeV} / c$ region by about one standard deviation and that above 10 $\mathrm{GeV} / \mathrm{c}$ by half its standard deviation. Having determined the neutral hadron background in the $5-10 \mathrm{GeV} / c$ region, one can use the observed momentum spectrum (Fig. 3) to calculate the expected background in the higher momentum regions. This calculation gives relatively large errors on the expected neutral hadron background because of the small number of associated events with momentum above $5 \mathrm{GeV} / c$.

There is also a possible systematic error associated with the criteria used to associate neutral-induced events with upstream events. If these criteria are too loose, some neutrino events will be incorrectly associated and the observed neutral hadron momentum spectrum will be too hard. If the criteria are too restrictive, some neutral hadron events will not be associated and the observed momentum spectrum may be too soft. We have checked these criteria in two ways. One is that associated events do not have EMI-identified muons and that they occur at the same time as the upstream event. The other involves frames which contain both a CC event and an $\mathrm{NC}$ candidate. There are 33 such frames where the $\mathrm{CC}$ event is upstream of the $\mathrm{NC}$ candidate and 34 frames where the CC event is downstream. The consistency of these two numbers also confirms the validity of the association criteria.

The expected hadron background for momentum above $25 \mathrm{GeV} / \mathrm{c}$ is slightly greater than the number of events vetoed by IPF $\mathrm{A}$ associations and thus justifies the assumption that any remaining contamination is negligible. (Using the calculated background would give $\mathrm{NC} / \mathrm{CC}=0.309$ instead of 0.323.) However, the expected hadron background above $10 \mathrm{GeV} / c$ is 174.1 events but only 72 events are vetoed by IPF A. Therefore, only $41 \%$ of the neutral hadron background for this momentum cut is removed using the IPF A veto.

Subtracting the expected backgrounds from the 742 $\mathrm{NC}$ candidates with visible longitudinal hadron momentum above $10 \mathrm{GeV} / c$ leaves an NC signal of 427.3 events. Correcting for losses and dividing by the corrected number of CC events (see Table III) gives a ratio NC/CC of $0.288 \pm 0.032$. The error is derived from only the statistical uncertainties in the numbers of observed events and in the data used to calculate backgrounds. To estimate possible systematic errors, we have examined in detail about half of the NC sample to look for other sources of contamination. For example, a muon which is mismeasured or mislabeled can cause a true CC event to be put in the $\mathrm{NC}$ sample. Also, a CC event which occurs before or after the EMI gate (i.e., the 4 msec time interval during which data is recorded for each beam spill) will 
contaminate the $\mathrm{NC}$ sample. In fact, nearly all of the possible systematic sources of error we have examined will tend to increase the observed ratio NC/CC. Based on the $\mathrm{CC}$ events recovered from the $\mathrm{NC}$ candidates examined in detail, we estimate that the systematic error on the value of $\mathrm{NC} / \mathrm{CC}$ to be at most 0.010 . This error could be reduced by completing the examination of the event sample but is already considerably smaller than the statistical error.

Our value of $\mathrm{NC} / \mathrm{CC}=0.288 \pm 0.032$ is for a combined sample of $\nu$ and $\bar{\nu}$ events but is mainly determined by the dominant $\nu$ component. If we assume an $\mathrm{NC} / \mathrm{CC}$ value for the $\bar{\nu}$ component, we can use the observed numbers of $\nu \mathrm{CC}$ and $\bar{\nu} \mathrm{CC}$ events to calculate an $\mathrm{NC} / \mathrm{CC}$ value for the $\nu$ component. The result is $\mathrm{NC} / \mathrm{CC}=0.274 \pm 0.038$ for the $\nu$ component assuming $\bar{\nu} \mathrm{NC} / \mathrm{CC}=0.39 \pm 0.08$. The $\bar{\nu}$ NC/CC value is based upon previous experiments, but the uncertainty is enlarged to allow for possible variation with energy.

\section{Comparison with other experiments}

Table IV compares our results with those from other experiments [7-10] where the mean event energy is about
$100 \mathrm{GeV}$. Because our value of $\mathrm{NC} / \mathrm{CC}$ is for a combined sample of $\nu$ and $\bar{\nu}$ events, we would expect to observe 0.32 if the ratios for $\nu$ and $\bar{\nu}$ are those observed by the CHARM [7] and CERN-Dortmund-HeidelbergSaclay (CDHS) [8] experiments. Our result is within one standard deviation of this expected value, consistent with $\mathrm{NC}$ interactions having the expected strength $\left(\sin ^{2} \theta_{W}=\right.$ 0.230 ) at neutrino energies up to $500 \mathrm{GeV}$. Our result also agrees with previous bubble-chamber experiments $[9,10]$ and with the Chicago-Columbia-FermilabRochester (CCFR) experiment [11]. The Big European Bubble Chamber (BEBC) experiment [9] imposes the additional requirement that the transverse momentum of the hadron system exceed $1 \mathrm{GeV} / c$. This requirement reduces the contamination of the NC sample by CC and by neutral hadron-induced events. Our backgrounds would be comparable to their values if we were to impose this same requirement.

\section{STRANGE-PARTICLE PRODUCTION}

An interesting aspect of $\mathrm{NC}$ interactions that cannot be studied with existing electronic detectors is strangeparticle production. Processes that produce leading

TABLE IV. Comparison of experiments which determine NC/CC at mean event energy around $100 \mathrm{GeV}$. For E632, only $70 \%$ of the total hadron energy is measured on average so requiring the measured hadron energy to be above $10 \mathrm{GeV}$ means that the true hadron energy is on average above $14 \mathrm{GeV}$.

\begin{tabular}{|c|c|c|c|c|c|c|c|c|}
\hline \multirow{3}{*}{ Minimum hadron energy } & \multicolumn{2}{|c|}{$\begin{array}{l}\text { BEBC }^{a} \\
\text { Ref. [9] }\end{array}$} & \multicolumn{2}{|c|}{$\begin{array}{c}\text { CHARM } \\
\text { Ref. [7] }\end{array}$} & \multicolumn{2}{|c|}{$\begin{array}{l}\text { CDHS } \\
\text { Ref. [8] }\end{array}$} & \multirow{3}{*}{$\begin{array}{c}\begin{array}{c}\text { E546 } \\
\text { Ref. [10] }\end{array} \\
10 \mathrm{GeV}\end{array}$} & \multirow{3}{*}{$\begin{array}{c}\mathrm{E} 632 \\
10 \mathrm{GeV}\end{array}$} \\
\hline & \multicolumn{2}{|c|}{$8 \mathrm{GeV}$} & \multicolumn{2}{|c|}{$4 \mathrm{GeV}$} & \multicolumn{2}{|c|}{$10 \mathrm{GeV}$} & & \\
\hline & $\nu$ & $\bar{\nu}$ & $\nu$ & $\bar{\nu}$ & $\nu$ & $\bar{\nu}$ & & \\
\hline Mean $\nu$ event energy $(\mathrm{GeV})$ & 103 & & 103 & & 103 & & 90 & 151 \\
\hline Mean $\bar{\nu}$ event energy $(\mathrm{GeV})$ & & 81 & & 81 & & 81 & 60 & 112 \\
\hline Identified $\mu^{-}$events & 2222 & & 104220 & & 132657 & & 1052 & 1492 \\
\hline Identified $\mu^{+}$events & & 653 & & 5005 & & 5228 & 195 & 249 \\
\hline Identified $\mathrm{e}^{+-}$events & 65 & $?$ & 0 & 0 & 0 & 0 & 35 & 37 \\
\hline CC loss fraction & 0.057 & 0.038 & 0.035 & 0.009 & 0.091 & 0.046 & 0.137 & 0.055 \\
\hline $\mathrm{NC}$ candidates & 956 & 288 & 36976 & 1947 & 56896 & 2374 & 587 & 670 \\
\hline CC background fraction & 0.105 & 0.077 & 0.149 & 0.052 & 0.205 & 0.120 & 0.374 & 0.210 \\
\hline Neutral hadron fraction & 0.038 & 0.033 & 0 & 0 & 0 & 0 & 0.066 & $0.152^{b}$ \\
\hline NC loss fraction & 0.011 & 0 & 0.051 & 0.053 & 0.003 & 0.003 & 0.204 & 0.074 \\
\hline $\mathrm{NC} / \mathrm{CC}$ & 0.345 & 0.364 & 0.3093 & 0.390 & 0.3072 & 0.382 & 0.301 & 0.288 \\
\hline Statistical error & 0.015 & 0.029 & 0.0031 & 0.014 & 0.0025 & 0.015 & 0.026 & 0.032 \\
\hline Systematic error & 0.009 & 0.009 & & & 0.0022 & 0.006 & & 0.010 \\
\hline NC/CC (9 GeV cut) & & & 0.3052 & 0.397 & & & & \\
\hline Error & & & 0.0033 & 0.015 & & & & \\
\hline
\end{tabular}

a Total hadron transverse momentum required to exceed $1 \mathrm{GeV} / c$ for $\mathrm{NC}$ sample.

${ }^{b}$ Neutral hadron background fraction without IPF A veto would be 0.235 . 
strange particles are different for $\mathrm{NC}$ and $\mathrm{CC}$ interactions at the quark level. In the standard model, an NC interaction with a strange quark will produce a leading strange quark, which, in the hadronization process, will usually form a strange particle that carries a substantial fraction of the total hadron energy. In CC interactions, a leading strange particle can result from the decay of a leading charm particle, which can be produced either from a down quark or from a strange quark. However, determining rates for production of leading strange quarks is complicated by processes in which a leading nonstrange quark picks up a strange antiquark in the hadronization process. ( $\mathrm{NC}$ interactions can produce either a leading down quark or a leading up quark; $\nu$ CC interactions produce primarily leading up quarks and $\bar{\nu} \mathrm{CC}$ interactions produce primarily leading down quarks.) The total strange-particle production rates and distributions will depend on quark densities and couplings, and on the strange-quark suppression factor and the relative fractions of pseudoscalar and vector mesons in the hadronization process. We will compare $K^{0}$ and $\Lambda$ production in $\mathrm{NC}$ and $\mathrm{CC}$ events (with the same cut on the total hadron momentum) both in overall rate and as a function of the fractional hadronic energy $z$ carried by the strange particle.

Neutral-strange-particle decays into two charged particles ( $V^{0}$ 's) that appear to be associated with the neutrino interaction vertex are measured as part of the event measurement. The kinematic fitting program SQUAW fits the measured $V^{0}$ 's to check for consistency with $K^{0}, \Lambda$, or $\bar{\Lambda}$ decays or with $\gamma$ conversions [one-constraint (1C) fits]. All $V^{0}$ 's which have successful $1 \mathrm{C}$ fits are then constrained ( $3 \mathrm{C}$ fits) to originate at the neutrino vertex. The $3 \mathrm{C}$ fit is accepted if the $\chi^{2}$ is less than 16 (confidence level $>0.001$ ). About $73 \%$ of $V^{0}$ 's with $1 \mathrm{C}$ fits have at least one acceptable $3 \mathrm{C}$ fit; about $\frac{1}{3}$ of these have more than one acceptable $3 \mathrm{C}$ fit. If both $K^{0}$ and $\Lambda$ fits are acceptable, the $\Lambda$ hypothesis is chosen unless the $\Lambda$-fit confidence level is below $4 \%$ and the $K^{0}$ confidence level is above $4 \%$. Ambiguities between $K^{0}$ and $\bar{\Lambda}$ fits are resolved by choosing the $K^{0}$ hypothesis unless the $K^{0}$ confidence level is below $4 \%$ and the $\bar{\Lambda}$ confidence level is above $4 \%$. To reduce biases, we require decays to occur at least $1 \mathrm{~cm}$ from the neutrino vertex and at least $20 \mathrm{~cm}$ from the bubble-chamber wall. Each $K^{0}$ or $\Lambda$ is then weighted by exponential factors to correct for losses due to very short or very long decay lengths and due to interaction before decay.

The observed numbers of $K^{0}, \Lambda$, and $\bar{\Lambda}$ in the CC and NC samples and the corresponding rates determined using this minimum-bias event sample are given in $\mathrm{Ta}$ ble $\mathrm{V}$. The corrected $K^{0}$ and $\Lambda$ rates include a detection and processing efficiency estimated to be $0.85 \pm 0.10$. The $K^{0}$ and $\Lambda$ rates are comparable in the NC and CC samples. Note that total strange-particle production in the CC sample increases with the cut on visible hadron momentum but that for the NC sample slightly decreases. This is consistent with the expectation that most of the neutral hadron contamination in the $\mathrm{NC}$ sample in the momentum range $10-25 \mathrm{GeV} / c$ is due to $K^{0}$-induced events. In fact, the observed $V^{0}$ rate in the 72 events ve-

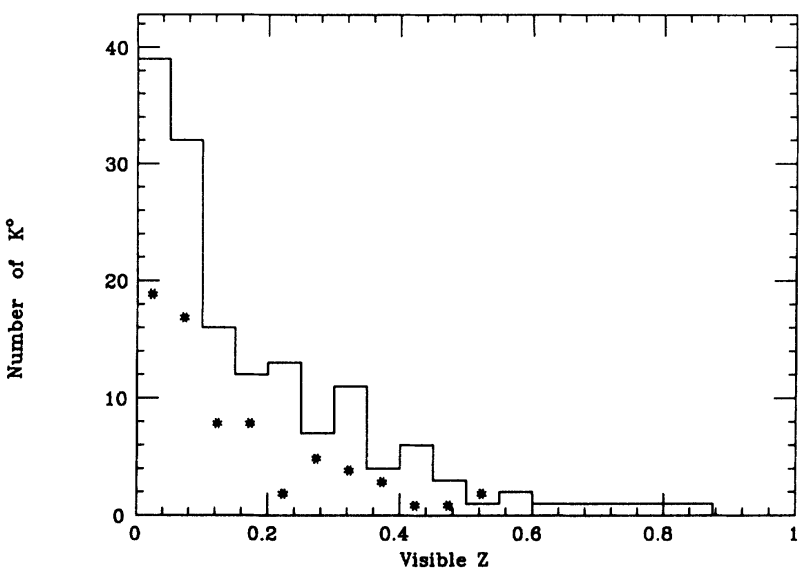

FIG. 6. $z$ distributions for $K^{-0}$ in $\mathrm{CC}$ and $\mathrm{NC}$ events for which the hadronic visible longitudinal momentum exceeds $25 \mathrm{GeV} / c$. The histogram is the data for $\nu$ CC plus $\bar{\nu} \mathrm{CC}$ events. The asterisks indicate the data for the NC sample.

toed by IPF $\mathrm{A}$ is $0.153 \pm 0.049$ compared to $0.121 \pm 0.014$ for the events remaining in the NC sample. Correcting for backgrounds in the NC sample, the observed $V^{0}$ rate for events with hadron momenta in the $10-25 \mathrm{GeV} / c$ region is $0.146 \pm 0.057$ for $\mathrm{NC}$ events compared to $0.078 \pm 0.014$ for CC events. This possible difference in the $V^{0}$ rates is statistically marginal and is not observed for events with larger hadron momenta.

In order to have more data for studies of the characteristics of strange-particle production [12], a sample of events with associated $V^{0}$ 's was measured in a portion of film which contains about $25 \%$ more events than the film from which the minimum bias sample comes. The numbers of $K^{0}, \Lambda$, and $\bar{\Lambda}$ observed in this additional $V^{0}$ event sample are given at the bottom of Table $V$. These events are not used in calculating production rates but are used in the $K^{0}$ and $\Lambda$ distributions. The distributions in the fractional hadronic energy $z$ for $K^{0}$ and $\Lambda$ for CC and NC samples with visible hadron momentum above $25 \mathrm{GeV} / c$ are shown in Figs. 6 and 7. (To avoid bias in the hadronic

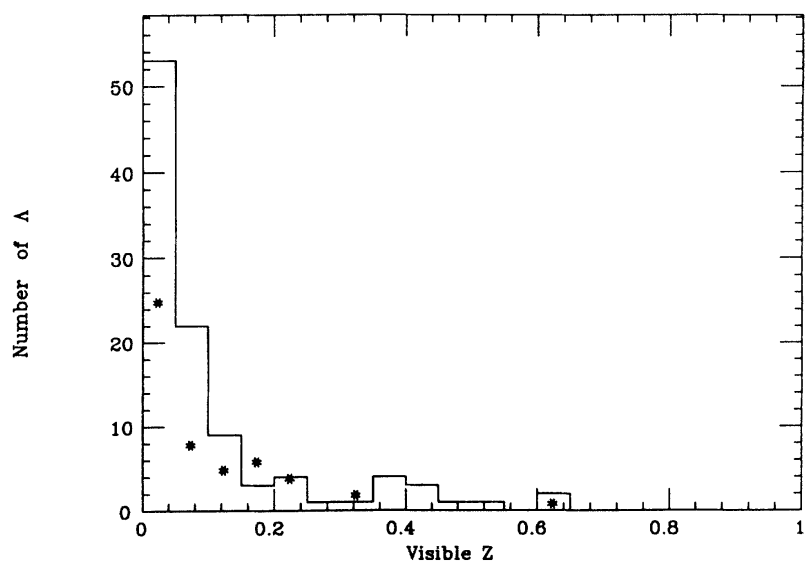

FIG. 7. $z$ distributions for $\Lambda$ in $\mathrm{CC}$ and $\mathrm{NC}$ events for which the hadronic visible longitudinal momentum exceeds $25 \mathrm{GeV} / c$. As in Fig. 6, the histogram is for $\nu$ CC plus $\bar{\nu}$ $\mathrm{CC}$ events and the asterisks are for the NC sample. 
energy due to fragments of the neon nucleus, we define $z$ as the magnitude of the particle's momentum divided by the magnitude of the total visible hadronic momentum.) These distributions are comparable for the $\mathrm{CC}$ and NC samples. Dividing the weighted numbers of $K^{0}$ 's in the $z>0.3$ region by the total numbers of $K^{0}$ 's gives $0.21 \pm 0.06$ for the NC sample, $0.27 \pm 0.05$ for $\nu \mathrm{CC}$ everits, and $0.39 \pm 0.11$ for $\bar{\nu}$ CC events.

Another aspect of the strange-particle event sample that deserves some mention is the observation of events with three or more associated $V^{0}$ 's. There is one $\mathrm{CC}$ event with three $V^{0}$ 's in the minimum bias sample. The additional $V^{0}$ event sample contains two $\mathrm{CC}$ and three $\mathrm{NC}$ events with three $V^{0}$ 's plus one $\mathrm{CC}$ and one $\mathrm{NC}$ event with four $V^{0}$ 's. The four CC events all have at least six tracks at the primary vertex and have visible hadron momenta ranging from 39 to $144 \mathrm{GeV} / c$. Based on the $\mathrm{CC}$ events, one would expect only $2.0 \mathrm{NC}$ candidates with three or more $V^{0}$ 's. Two of the four NC candidates have seven and six primary tracks, respectively, with visible

TABLE V. Numbers of observed neutral strange particles and production rates in CC and NC events for different regions of visible hadron momentum. The corrected rates include corrections for branching ratios into two charged particles as well as corrections for losses at very short and long decay lengths, losses due to interaction before decay, and detection-processing losses.

\begin{tabular}{lcr}
\hline \hline Visible long. hadron momentum & $>10 \mathrm{GeV} / c$ & $>25 \mathrm{GeV} /$ \\
\hline Total CC events & 1358 & 911 \\
& & \\
Observed $\mathrm{K}^{0}$ & 85 & 68 \\
Observed $\Lambda$ & 70 & 50 \\
Observed $\bar{\Lambda}$ & 6 & 4
\end{tabular}

Rate for observed $\mathrm{V}^{0}$ 's

Corrected $K^{0}$ rate

Corrected $\Lambda$ rate

Total NC events

Observed $K^{0}$

Observed $\Lambda$

Observed $\bar{\Lambda}$

Rate for observed $V^{0}$ 's

Rate in events with

associated IPF A hits

Corrected $K^{0}$ rate

Corrected $\Lambda$ rate
$0.119 \pm 0.010$

$0.309 \pm 0.050$

$0.141 \pm 0.023$

670

\section{8}

29

4

$0.121 \pm 0.014$

$0.153 \pm 0.049$

$0.344 \pm 0.065$

$0.111 \pm 0.025$
$0.134 \pm 0.013$

$0.375 \pm 0.064$

$0.161 \pm 0.030$

437

28

19

2

$0.112 \pm 0.017$

$0.095 \pm 0.070$

$0.322 \pm 0.073$

$0.113 \pm 0.030$

Data below from film in which only events with V's were measured

Observed $K^{0}$ in $\mathrm{CC}+\mathrm{V}$ events

Observed $\Lambda$

Observed $\bar{\Lambda}$
81

55

4

41

30

$\begin{array}{cc}43 & 30 \\ 3 & 3\end{array}$


hadron momenta of 25 and $43 \mathrm{GeV} / c$, but the other two $\mathrm{NC}$ candidates have only one track at the primary vertex. These 1-prong events both have two $K^{0}$ 's and one $\Lambda$ and total visible momenta of 21 and $28 \mathrm{GeV} / c$. Only two of the $670 \mathrm{NC}$ events in the minimum bias sample are 1 -prong events and neither event has an associated $V^{0}$.

\section{SUMMARY}

Using an improved EMI and IPF in conjunction with the Fermilab 15-foot bubble chamber exposed to the Tevatron quadrupole triplet neutrino beam, we have extracted a sample of high energy NC events with backgrounds and losses which are smaller than or comparable to previous experiments. For visible longitudinal hadronic momentum above $10 \mathrm{GeV} / c$, we find $\mathrm{NC} / \mathrm{CC}$ $=0.288 \pm 0.032$ for an average $\nu(\bar{\nu})$ event energy of 150 (110) $\mathrm{GeV}$. Assuming that the smaller $\bar{\nu}$ component has $\mathrm{NC} / \mathrm{CC}=0.39 \pm 0.08$, we find $\mathrm{NC} / \mathrm{CC}=0.274 \pm 0.038$ for $\nu$ events, which comprise about $88 \%$ of all CC events with visible longitudinal hadronic momentum above 10 $\mathrm{GeV} / c$. The value of $\mathrm{NC} / \mathrm{CC}$ is $0.323 \pm 0.025$ for the combined $\nu$ and $\bar{\nu}$ sample with visible hadronic momentum above $25 \mathrm{GeV} / c$. Possible sources of systematic errors would result in an overestimate of $\mathrm{NC} / \mathrm{CC}$ by at most 0.010 . Our NC/CC value agrees with those determined by previous experiments. Production rates and $z$ distributions for $K^{0}$ and $\Lambda$ particles in NC events are consistent with those in $\mathrm{CC}$ events.

\section{ACKNOWLEDGMENTS}

There are many people who made important contributions before and during the E632 runs but did not participate in this analysis. We acknowledge the assistance of the Fermilab staff and especially the crew of the 15-foot bubble chamber. Because the IPF and EMI are crucial for this analysis, we also acknowledge Sten Hansen and Stephen Pordes (Fermilab) and Dan Chapman (U.C. Berkeley), whose efforts contributed to the excellent performance of these devices. We also acknowledge our colleagues at Rutherford Appleton Lab and the scanning and measuring staffs at the participating institutions. This work was supported in part by U.S. Department of Energy Contracts DE-AC03-83ER40103, DE-AC0283ER40085, and DE-AC02-76CH03000, by National Science Foundation Grants PHY89-07526, PHY88-17930, and PHY89-06760 and by S.E.R.C. Grant GR/G 00938 in the U.K. P.P.A., P.N., and S. Wainstein were supported by the U.K.S.E.R.C.

\section{APPENDIX: DETERMINING EVENT TIME SLOTS USING THE IPF}

A new feature of this analysis is the use of the Internal Picket Fence (IPF) to determine the time slot for each event. (A time slot is a $1-\mu$ sec interval within the $4 \mathrm{msec}$ during which data from the IPF and EMI is recorded for each beam spill, whose typical duration was 2 to $3 \mathrm{msec}$.) Determination of an event's time slot is important in order to reject neutral hadron-induced events and to reduce losses from the NC sample due to hadron misidentification. Charged particles associated with an event can make hits in the IPF tubes which are just outside the bubble-chamber wall. Because most particles are produced at small angles with respect to the incident neutrino direction, most of the IPF tubes hit will be in the downstream half (IPF B). Usually there will be no hits in the upstream half (IPF A) unless the event is induced by a neutral hadron which was produced, along with several charged particles, upstream of the chamber. Therefore, one can determine an event's time slot by looking for a time slot in which there are many IPF $\mathrm{B}$ tubes with hits; neutral hadron-induced events will usually have many IPF A tubes with hits and thus can be rejected.

The determination of an event's time slot proceeds as follows. Given the $X$ and $Y$ coordinates of the event vertex, one calculates a range of tubes in IPF $B$ within which there are likely to be hits. This range is defined by a cone of half-angle of 120 milliradians whose apex is at the event vertex and whose axis is along the incident neutrino beam direction. The intersection of this cone with IPF $B$ determines a range of tubes within which to look for hits. The intersection of this cone with IPF A defines a range of tubes upstream of the event within which to look for hits. The choice of the cone angle was made by looking at the distribution of azimuthal angles of muons from $\mathrm{CC}$ events and of neutral hadron-induced events associated with upstream neutrino interactions. Note that the ranges of tubes are determined only by the location of the event and do not depend on any other properties.

Many events will have measured tracks which leave the chamber without interacting and which are predicted to hit tubes in IPF B. For such events, one also counts the number of predicted tubes which have hits for each time slot. (There are two layers of tubes in the IPF so a single charged particle usually causes two adjacent tubes to be hit.) For each time slot, three quantities are calculated:

\footnotetext{
$B_{\text {mat }}=$ number of predicted IPF $\mathrm{B}$ tubes that have hits,

$A_{\text {hit }}=$ number of IPF A tubes inside cone that have hits,

$B_{\text {hit }}=$ number of IPF $\mathrm{B}$ tubes inside cone that have hits + number of predicted tubes outside the cone that have hits.
} 
Most event time slots will have large values of $B_{\text {hit }}$ whether or not there are measured leaving tracks. The second term in the calculation of $B_{\text {hit }}$ is included to allow for events in which there are several tracks which bend so much that they hit IPF B outside the cone.

In most cases there is only one time slot for which $B_{\text {hit }}$ is at least 3 ; this time slot is chosen as the event time slot unless the event has leaving tracks and none of the predicted tubes has a hit. If there are no time slots which satisfy these criteria, another iteration is done where the cone angle used to determine the range in IPF $B$ is tripled. If there are still no time slots which satisfy the criteria, a final iteration is done including all of IPF B. There are three possible cases: (I) unique time slot with $B_{\text {hit }}$ at least 3 ; (II) no time slots with $B_{\text {hit }}$ at least 3 ; (III) more than one time slot with $B_{\text {hit }}$ at least 3 .

In case I, the unique time slot is chosen. In case II, the time slot with the largest value of $B_{\text {hit }}$ is chosen if there are no predicted tubes. If there are predicted tubes, the time slot with the largest value of $B_{\text {mat }}$ is chosen. The selection will not be very reliable because any beam tracks near the event will produce time slots with $B_{\text {hit }}$ $=2$. If the beam track density is high, it is likely that a beam-track time slot will be chosen instead of the event time slot. The most difficult case is III. Probably the best way to decide such cases would be to measure as many leaving tracks as possible and pick the time slot with the most matches. Unfortunately, many NC events have no measured leaving tracks. Therefore, the choice of time slot depends upon the number of predicted tubes. If there are at least 4 IPF $B$ tubes predicted to be hit, the time slot with the largest $B_{\text {mat }}$ value is chosen. If there are 2 or 3 IPF $B$ tubes predicted to be hit, we choose the time slot with some IPF B matches. If there is no unique time slot which satisfies the criteria in these cases or, if there are 0 or 1 IPF $B$ tubes predicted to be hit, we use criteria based on the values of $B_{\text {hit }}$. If the total number of hits is less than 10 , we pick the time slot with the largest $B_{\text {hit }}$ value. If there are at least two time slots with 10 or more total hits, we choose the one for which $B_{\text {hit }}$ is the largest fraction of the total.

One can estimate the efficiency of this technique to choose the correct time slot by using CC events and comparing with the time slot determined by the EMI for the muon. (Studies of muon identification using the EMI indicate that less than $1 \%$ of EMI-identified muons correspond to a time slot different from the event time slot.) This efficiency depends upon the hadronic energy because most hits in IPF $B$ will be due to hadrons or to electrons from conversions of gamma rays. Therefore, we calculate efficiencies for three different intervals of visible (measured) hadron momentum:

$$
\begin{array}{rlrl}
5-10 \mathrm{GeV} / c, \text { efficiency } & =133 / 184=0.72 \pm 0.03 \\
& =10 \mathrm{GeV} / c, & & 1245 / 1359=0.92 \pm 0.01 \\
>25 \mathrm{GeV} / c, & & 859 / 915=0.94 \pm 0.01
\end{array}
$$

In the sample of $1359 \mathrm{CC}$ events with visible hadron momentum above $10 \mathrm{GeV} / c$, the average value of $B_{\text {hit }}$ for the selected event time slot is 18 but only 5 time slots have 3 or more IPF A tubes with hits within the cone upstream of the event.

Therefore, rejecting events with $A_{\text {hit }}$ greater than or equal to 3 will exclude only $5 / 1359=0.0037$ of neutrinoinduced events with visible hadron momentum above 10 $\mathrm{GeV} / c$. (None of the 1245 events for which the selected event time slot is the same as the muon time slot is rejected by this requirement.)
[1] U. Amaldi et al., Phys. Rev. D 36, 1385 (1987).

[2] K. Winter, in Neutrino '88, Proceedings of the 13th International Conference on Neutrino Physics and Astrophysics, Boston, Massachusetts, 1988, edited by J. Schneps et al. (World Scientific, Singapore, 1989), p. 403.

[3] G. Costa et al., Nucl. Phys. B297, 244 (1988).

[4] G. L. Fogli and D. Haidt, Z. Phys. C 40, 379 (1988).

[5] R. Barbieri, in Proceedings of the International Europhysics Conference on High Energy Physics, Madrid, Spain, 1989, edited by F. Barreiro and C. Lopez [Nucl. Phys. B (Proc. Suppl.) 16 (1990)].
[6] V. Jain et al., Phys. Rev. D 41, 2057 (1990).

[7] J. V. Allaby et al., Z. Phys. C 36, 611 (1987).

[8] A. Blondel et al., Z. Phys. C 45, 361 (1990).

[9] P. C. Bosetti et al., Nucl. Phys. B217, 1 (1983); H. Deden et al., Phys. Lett. 98B, 310 (1981).

[10] M. D. Jones et al., Report No. UH-511-531-84, presented to the 11th International Symposium on Neutrino Physics and Astrophysics, Dortmund, West Germany, 1984 (unpublished).

[11] P. G. Reutens et al., Z. Phys. C 45, 539 (1990).

[12] D. DeProspo, Ph.D. thesis, Rutgers University, 1991. 